\title{
Article \\ Pharmacological Modulation of Behaviour, Serotonin and Dopamine Levels in Daphnia magna Exposed to the Monoamine Oxidase Inhibitor Deprenyl
}

\author{
Marina Bellot $^{1}\left(\mathbb{D}\right.$, Melissa Faria ${ }^{2}$, Cristian Gómez-Canela ${ }^{1} \mathbb{D}$, Demetrio Raldúa ${ }^{2}$ (D) and Carlos Barata ${ }^{2, *}$ \\ 1 Department of Analytical Chemistry and Applied (Chromatography Section), School of Engineering, \\ Institut Químic de Sarrià-Universitat Ramon Llull, Via Augusta 390, 08017 Barcelona, Spain; \\ marina.bellot@iqs.url.edu (M.B.); cristian.gomez@iqs.url.edu (C.G.-C.) \\ 2 Institute for Environmental Assessment and Water Research (IDAEA-CSIC), Jordi Girona 18, \\ 08034 Barcelona, Spain; mdfqam@cid.csic.es (M.F.); drpqam@cid.csic.es (D.R.) \\ * Correspondence: carlos.barata@idaea.csic.es
}

Citation: Bellot, M.; Faria, M.; Gómez-Canela, C.; Raldúa, D.; Barata, C. Pharmacological Modulation of Behaviour, Serotonin and Dopamine Levels in Daphnia magna Exposed to the Monoamine Oxidase Inhibitor Deprenyl. Toxics 2021, 9, 187. https://doi.org/10.3390/toxics9080187

Academic Editor: Ki-Tae Kim

Received: 15 July 2021

Accepted: 6 August 2021

Published: 9 August 2021

Publisher's Note: MDPI stays neutral with regard to jurisdictional claims in published maps and institutional affiliations.

Copyright: (c) 2021 by the authors. Licensee MDPI, Basel, Switzerland. This article is an open access article distributed under the terms and conditions of the Creative Commons Attribution (CC BY) license (https:/ / creativecommons.org/licenses/by/ $4.0 /)$.

\begin{abstract}
This study assessed the effects of the monoamine oxidase (MAO) inhibitor deprenyl in Daphnia magna locomotor activity. The mechanisms of action of deprenyl were also determined by studying the relationship between behaviour, MAO activity and neurotransmitter levels. Modulation of the D. magna monoamine system was accomplished by $24 \mathrm{~h}$ exposure to two model psychotropic pharmaceuticals with antagonistic and agonistic serotonin signalling properties: $10 \mathrm{mg} / \mathrm{L}$ of 4-chloroDL-phenylalanine (PCPA) and $1 \mathrm{mg} / \mathrm{L}$ of deprenyl, respectively. Contrasting behavioural outcomes were observed for deprenyl and PCPA reflected in decreased basal locomotor activity and enhanced habituation for the former compound and delayed habituation for the latter one. Deprenyl exposure inhibited monoamine oxidase (MAO) activity and increased the concentrations of serotonin, dopamine and the dopamine metabolite 3-methoxytyramine in whole D. magna extracts. Our findings indicate that D. magna is a sensitive and useful nonvertebrate model for assessing the effects of short-term exposure to chemicals that alter monoamine signalling changes.
\end{abstract}

Keywords: Daphnia magna; neurotransmitter; modulation; pharmaceuticals

\section{Introduction}

Animal behaviour to environmental stimuli changes such as predation or food availability is a key fitness trait [1]. Of particular interest are behavioural responses related to predator avoidance such as sudden locomotion changes in response to visual or tactile stimuli [2,3]. Any changes in the normal behavioural conduct could compromise individual survival. Neurotransmitters modulate behavioural plasticity at the molecular level. Serotonin is one of the major neurotransmitters in the central nervous system (CNS), modulating many behaviours including perception, mood, reward, anger, aggression, appetite, memory, sexuality and attention [4]. Neurological pathologies such as schizophrenia, depression and anxiety have been related to dysfunctions in the serotonergic system. The serotonergic system is well-known in vertebrates, but little is known about many invertebrates. Tryptophan hydroxylase (TPH) is the enzyme that converts tryptophan to 5-hydroxytryptophan (5-HTP), which is the rate-limiting step of serotonin synthesis. When serotonin is released to the synaptic cleft, the serotonin transporter SERT mediates its uptake/reuptake to the serotonergic neurons, where monoamine oxidase (MAO) metabolizes serotonin to 5-hydroxy-indolecetaldehyde, which is quickly metabolized by aldehyde dehydrogenase to form 5-hydroxyindoleacetic acid (5-HIAA), the major metabolite of serotonin [5].

Many of the emerging contaminants present in surface waters are neuroactive substances required for highly prescribed drugs to control cardiac and neurological disorders [6]. Neuroactive compounds targeting the serotonergic system are of particular 
concern due to the essential role of serotonin in both neurotransmission and neuromodulation. Thus, it is not surprising to find many investigations on the effects of selective serotonin reuptake inhibitors (SSRIs) in nontarget aquatic species like fish but also in invertebrates $[7,8]$. Nevertheless, information regarding other potential modes of actions, such as TPH or monoamine oxidase (MAO) inhibition, is still scarce and almost absent for many invertebrates. In this regard, the ecotoxicological model species Daphnia magna is a good candidate for studying the role of serotonergic signalling pathways in behaviour and its modulation by neuroactive pollutants. D. magna is probably the most widely use organism in aquatic toxicological evaluations. It is a well-known ecological model and has its genome fully sequenced and annotated [9]. Therefore, D. magna offers the opportunity to study molecular and apical effects of pharmaceuticals. Furthermore, about $80 \%$ of the molecular human drug targets are also present in the Daphnia genome [10]. This means that it is likely that many neuroactive pollutants affect this organism. Recently, it was shown that CRISPR/Cas9-mediated tryptophan hydroxylase (TPH) knockout clonal D. magna lines lack serotonin and show an abnormally high basal swimming activity and a markedly reduced habituation to repetitive light stimuli [2,11]. The selective serotonin reuptake inhibitor (SSRI) fluoxetine that is the active ingredient of Prozac increases the serotonin levels in the brain of D. magna and increases reproduction [12]. Conversely, chloro-DL-phenylalanine (PCPA), which is an inhibitor of $\mathrm{TPH}$, decreases serotonin levels in D. magna [13]. Fluoxetine and PCPA, however, despite having opposite effects on serotonin levels, have similar effects on Daphnia cognitive behaviour, decreasing habituation to repetitive light stimuli [2]. The aim of this study was to better characterize the serotonergic system in Daphnia. In particular, we studied the mechanisms of action of monoamine oxidase (MAO) inhibitors, such as deprenyl, in D. magna, addressing its effects on the target MAO enzyme activity, neurotransmitter levels and behavioural responses. Our hypothesis is that deprenyl should increase serotonin and probably also dopamine levels in D. magna and have behavioural responses different from those of the drugs that depress serotonin levels, such as PCPA.

\section{Materials and Methods}

\subsection{Experimental Animals}

Five-day old juveniles from a single clone of D. magna (clone F) were used for exposure, behavioural and biochemical determinations assays. Further details of culture conditions are provided in the Supplementary Materials, Section S1.1.

\subsection{Experimental Procedures}

Deprenyl (CAS:14611-52-0) and chloro-DL-phenylalanine (PCPA; CAS: 7424-00-2) were of high-quality grade and purchased from Sigma-Aldrich (St. Louis, MO, USA). Stock solutions were prepared in the Milli-Q water and then diluted in ASTM hard water. D. magna juveniles (5-days-old) were pre-exposed to the selected compounds in ASTM hard water without food for $24 \mathrm{~h}$. The selected chemicals concentrations were far below those having detrimental effects on survival or swimming (>20 mg/L; [2]). The compounds were initially screened for light stimuli motile responses using a broad concentration range ranking from 0.1 to $1000 \mu \mathrm{g} / \mathrm{L}$ for deprenyl and from 0.1 to $10,000 \mu \mathrm{g} / \mathrm{L}$ for PCPA [2]. The concentrations having the greatest effect $(1$ and $10 \mathrm{mg} / \mathrm{L}$ for deprenyl and PCPA, respectively) were used in the subsequent light stimuli motile response assays. For the behavioural assessment, exposures were conducted in groups of 12 individuals in $300 \mathrm{~mL}$ of the test medium in $500 \mathrm{~mL}$ glass vessels. Following $24 \mathrm{~h}$ of exposure, 12 animals were distributed randomly to 24 -well plates (two treatments per plate). From 12 to 24 individual replicates were performed for each tested chemical. For the MAO activity and neurotransmitters assessment, exposures were carried out in groups of 20 or 5 individuals in $500 \mathrm{~mL}$ or $100 \mathrm{~mL}$ of the medium, respectively. The treatments were replicated five times. Following exposure, the individuals from each replicate (20 or 5) were pooled in an Eppendorf, the water was removed and the rest was deep-frozen in liquid $\mathrm{N}_{2}$. The samples were stored at 
$-80{ }^{\circ} \mathrm{C}$ until analysis. For each behavioural assay and MAO determination, juveniles were collected from 2-4 trials of the same experimental setup conducted on different days and with different batches of animals.

\subsection{Behavioural Analysis}

The Daphnia photomotor response assay (DPRA) was performed as described in [2]. Details of the assay are provided in the Supplementary Materials, Methods, Section S1.2. The assay measured the distance moved after a sudden increase in light intensity across 30 repetitive light stimuli of $1 \mathrm{~s}$ followed by $4 \mathrm{~s}$ of darkness. Following a previous study, "enhanced photomotor response" (EPR) is defined as the area under the curve (AUCEPR) for the first 10 stimuli where the response to light increases. Conversely, "habituation or non-associative learning" is defined as the area under the curve (AUCh) for the decreasing responses to stimuli [2].

To better characterize the swimming activity under darkness and upon continuous light, basal locomotor activity (BLM) and visual motor response (VMR) analyses of 5-daysold D. magna juveniles were also assessed using the same DanioVision system device as described below. Before the video recording, the juveniles were first acclimated for $10 \mathrm{~min}$ under dark conditions. Video tracking trials consisted of a 10 min cycle with a 5 min dark period followed by a $5 \mathrm{~min}$ light period (290 lux). The basal locomotor activity (BLM) was defined as the total distance $(\mathrm{mm})$ travelled by the juveniles during the last minute of the first dark cycle. The visual motor response (VMR) was based in the hyperactivity period induced by light, which in D. magna increased during the first minutes and decreased afterwards.

\subsection{Daphnia Monoamine Oxidase (MAO) Activity}

D. magna juveniles were collected in pools of 20 individuals and homogenized in ice-cold $10 \mathrm{mM}$ phosphate buffer ( $\mathrm{pH}$ 7.6) supplemented with $1 \mathrm{mM}$ EDTA using a TissueLyser ${ }^{\circledR}$ (Qiagen, Germantown, MA, USA). The volume of the buffer was adjusted to 100 juveniles $/ \mathrm{mL}$. Following centrifugation at $2500 \times \mathrm{g}, 4^{\circ} \mathrm{C}$ for $5 \mathrm{~min}, \mathrm{MAO}$ activity was determined in the supernatant according to Faria et al. [14]. Further information is provided in the Supplementary Materials, Section S1.3.

\subsection{Extraction and Analysis of Neurotransmitters}

Monoaminergic neurotransmitters were extracted from pools of five juveniles according to the procedure adapted from the article by Fuertes et al. [13]. Additional details on the extraction and analysis of neurotransmitters are provided in the Supplementary Materials, Section S1.4.

\subsection{Statistical Analysis}

The experimental design for behavioural assay responses of controls were compared to those of treatments using either the Student's $t$-test when only controls and deprenyl treatments were used or one-way ANOVA followed by Dunnett's post-hoc test when PCPA was also considered. MAO activities and concentration of metabolites of the unexposed juveniles and the deprenyl-treated ones were compared using the Student's $t$-test. For the Daphnia photomotor responses, the area under the curve $A U C_{E P R}$ and $A U C_{h}$ values obtained for the individual juveniles across the repetitive light stimuli were used for statistical comparisons. The basal and visual locomotor activities of the D. magna juveniles monitored during $5 \mathrm{~min}$ of dark and $5 \mathrm{~min}$ of light were analysed simultaneously using repeated measures ANOVA considering the total distance moved in the last minute of darkness and in each of the five minutes of the light period as the repeated measures (hereafter referred to as the "time"). Prior to the analyses, ANOVA assumptions of data normality and variance homoscedasticity were tested. Analysis of the data was performed with IBM SPSS v25 (Statistical Package 2010, Chicago, IL, USA). Significance was set at $p<0.05$. 


\section{Results}

\subsection{Behaviour}

For the Daphnia photomotor response assay (DPRA) to repetitive light stimuli, absolute and proportional distances moved across the tested compounds are reported in Figure 1 and the statistics are referred to the AUC values reported in the graph inlet. The statistics are depicted in Table 1. Deprenyl increased habituation significantly $(p<0.05)$ in three out of the four experiments (Figure 1A,B,D) and enhanced photomotor responses in only one of them (Figure 1D). PCPA reduced habituation in the two experiments that were performed and did not enhance photomotor responses (Figure 1C,D).
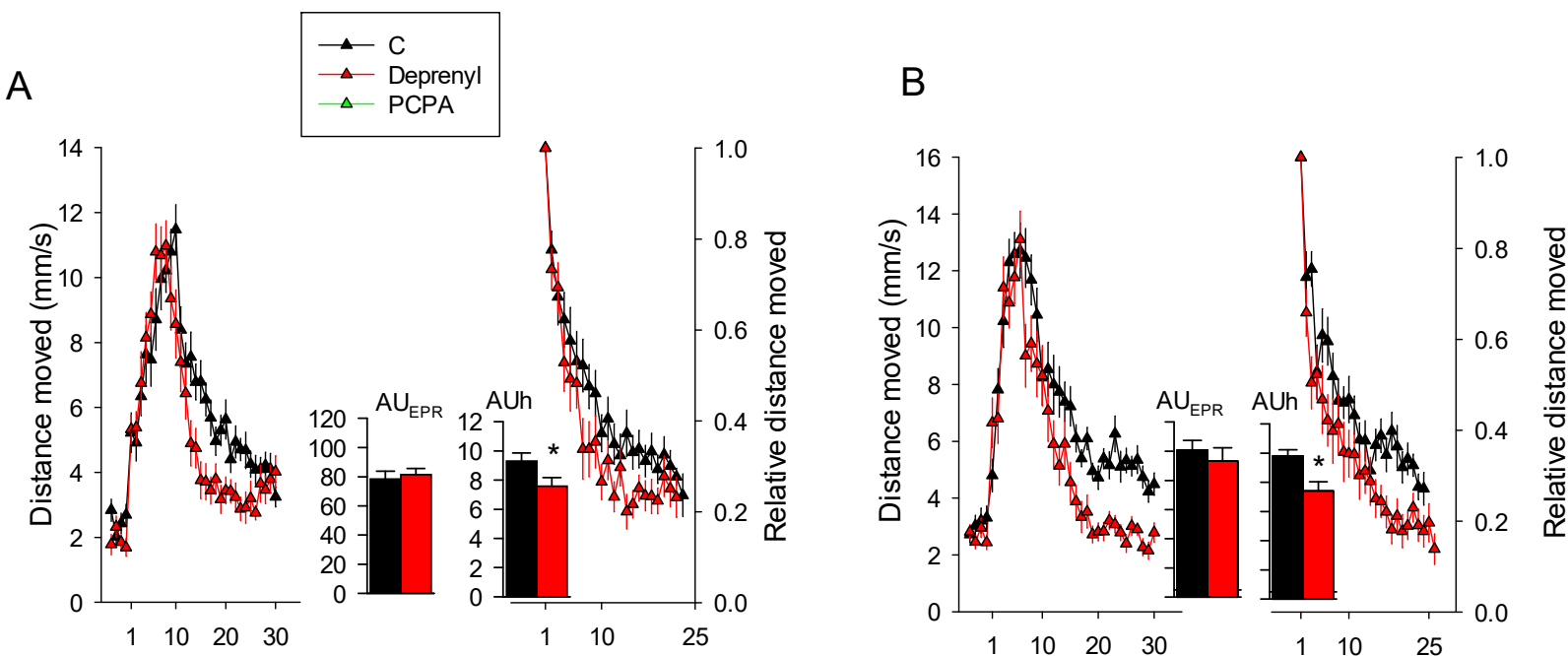

C
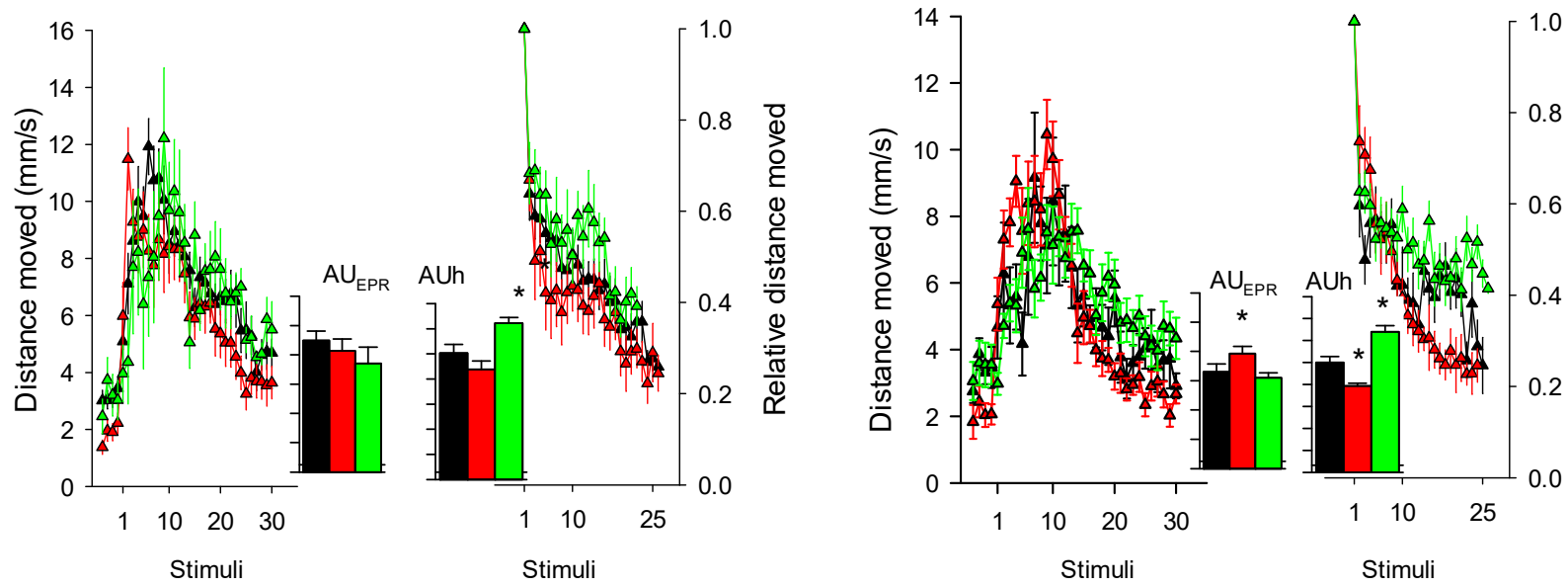

0
0
0
0
0
0
$\frac{1}{\pi}$
$\frac{0}{0}$
$\frac{0}{0}$
0
$\stackrel{D}{0}$
$\frac{\pi}{0}$
$\square$

Figure 1. Daphnia photomotor responses to repetitive light stimuli following $24 \mathrm{~h}$ exposures to 1 and $10 \mathrm{mg} / \mathrm{L}$ of deprenyl and PCPA, respectively. Plots of the average distance moved \pm SE $(n=12-24)$ against 30 tapping stimuli at $5 \mathrm{~s}$ ISI and

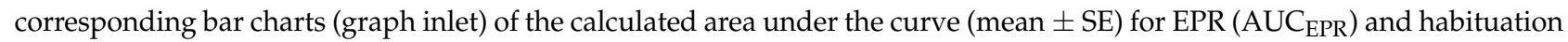
AUCh phases. Within each of the four graphs, the left plots represent the full motile responses whereas the right ones show habituation responses measuring the decrease in the distance moved (proportions) relative to the maximum response to the light stimulus delivered (set to 1). Graphs (A,B) depict the data obtained across two independent experiments for deprenyl exposures, (C,D)—for deprenyl and PCPA exposures. Within the AUC bar graphs, * means significant $(p<0.05)$ treatment differeces relative to the controls following the Student's $t$-test or ANOVA and Dunnett's test. Axis scales for the AUC (graph inlet) are depicted in graph (A). 
Table 1. Student's $t$-test or one-way ANOVA results testing the effects of deprenyl and PCPA on the area under the curve (AUC) values obtained from Daphnia photomotor responses to repetitive light stimuli. AUCEPR and AUCh are, respectively, the areas of enhanced photomotor responses during the first 10 light stimuli and during habituation afterwards. Results for the four identical experiments are reported.

\begin{tabular}{ccccc}
\hline & & df & t,F & $P$ \\
\hline Experiment 1 & AUCh & 31 & 2.1 & 0.047 \\
& AUCEPR & 31 & 0.4 & 0.657 \\
Experiment 2 & AUCh & 31 & 3.3 & 0.003 \\
& AUCEPR & 31 & 0.7 & 0.493 \\
Experiment 3 & AUCh & 2,45 & 10.2 & $<0.001$ \\
& AUCEPR & 2,45 & 0.8 & 0.459 \\
Experiment 4 & AUCh & 2,56 & 10.0 & $<0.001$ \\
& AUCEPR & 2,56 & 3.9 & 0.026 \\
\hline
\end{tabular}

Basal locomotor activity (BLM) and visual motor response (VMR) analyses of 5-dayold D. magna juveniles are depicted in Figure 2. Repeated measures ANOVA denoted significant $(p<0.05)$ effects of treatment or of its interaction with time (Table 2). In all the experiments, deprenyl decreased the basal locomotor activity and in only half of them (Figure 2A,C) increased the response to light. PCPA decreased the response to light in the two experiments performed (Figure 2C,D) and increased the basal locomotor activity in one of them (Figure 2C).
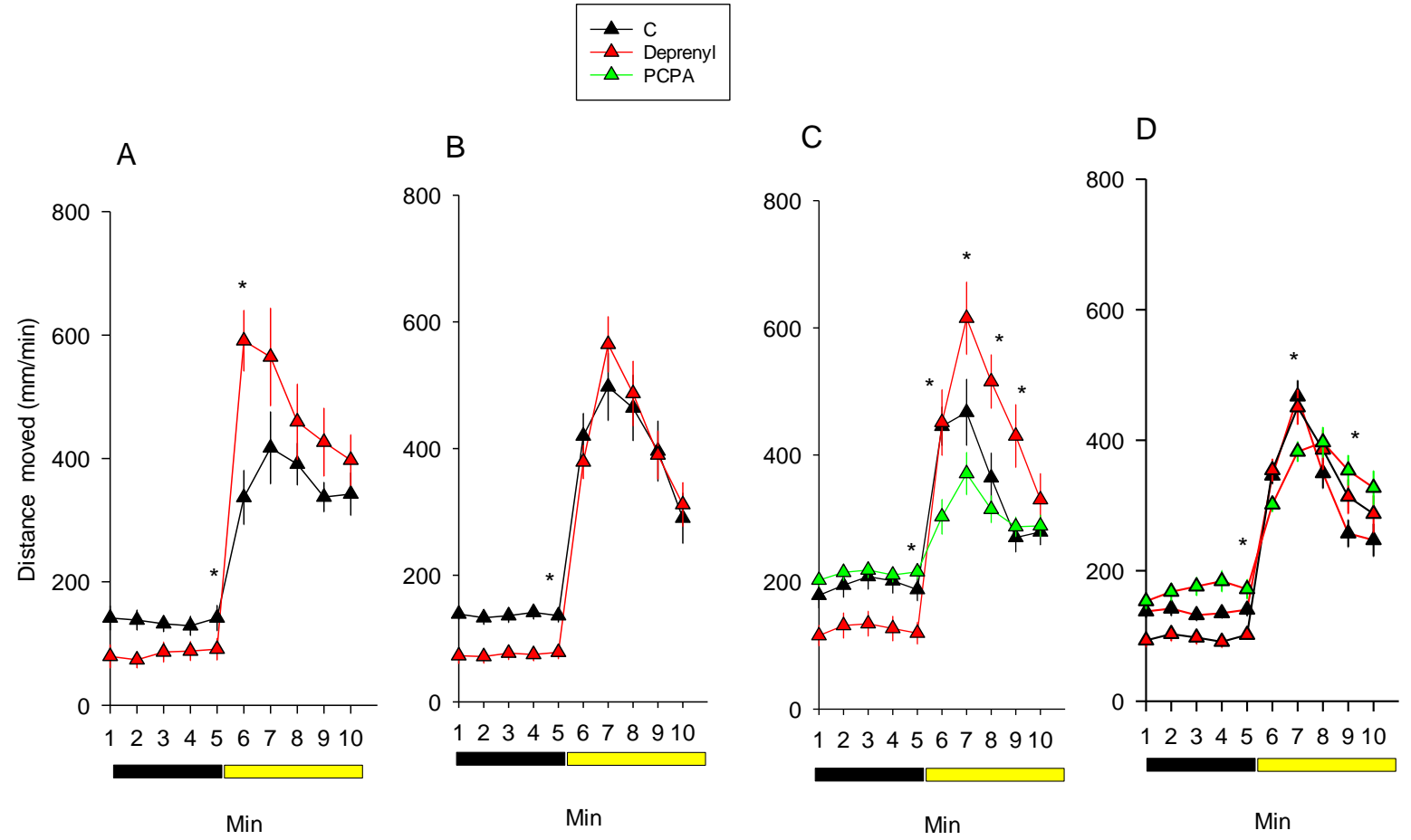

Figure 2. Daphnia basal locomotor activity (BLM) and visual motor responses (VMR) across 5 min in darkness and 5 min with light following $24 \mathrm{~h}$ exposures to 1 and $10 \mathrm{mg} / \mathrm{L}$ of deprenyl and PCPA, respectively. Plots of the average distance moved \pm SE $(n=12-24)$ per minute across the $10 \mathrm{~min}$ of the monitored period are shown. Graphs $(\mathbf{A}, \mathbf{B})$ depict the data obtained across two independent experiments for deprenyl exposures and (C,D) for deprenyl and PCPA ones. Note: * during the minutes $5-10$, mean significant $(p<0.05)$ treatment differences relative to the controls following the Student's $t$-test or ANOVA and Dunnett's test. 
Table 2. Results of repeated measures one-way ANOVA testing for the effects of treatment, monitoring time and its interaction (Time * Treatment) on the D. magna visual responses across the last minute of darkness and the 5 min of light. Results for four identical experiments are reported.

\begin{tabular}{ccccc}
\hline & & df & F & P \\
\hline Experiment 1 & Time & 1,22 & 16.6 & 0.001 \\
& Treatment & 1,22 & 4.4 & 0.048 \\
& Time * Treatment & 1,22 & 0.0 & 0.923 \\
Experiment 2 & Time & 1,46 & 12.6 & 0.001 \\
& Treatment & 1,46 & 0.2 & 0.688 \\
Experiment 3 & Time * Treatment & 1,46 & 4.2 & 0.047 \\
& Time & 1,69 & 10.9 & 0.002 \\
& Treatment & 2,69 & 8.7 & $<0.001$ \\
Experiment 4 & Time * Treatment & 2,69 & 5.7 & 0.005 \\
& Time & 1,68 & 17.9 & $<0.001$ \\
& Treatment & 2,68 & 3.9 & 0.026 \\
& Time * Treatment & 2,68 & 4.3 & 0.017 \\
\hline
\end{tabular}

\subsection{Biochemical and Neurotransmitter Analysis}

MAO activity was significantly $(p<0.05)$ inhibited by deprenyl in the three experiments performed (Figure $3 \mathrm{~A})$, and deprenyl significantly $(p<0.05)$ increased serotonin (5-HT), dopamine (DA) and 3-methoxytyramine (3-MT) (Figure 3B-D). Details of the statistics and of nonsignificant metabolite values are provided in Tables S3 and S4, respectively, Supplementary Materials.

A
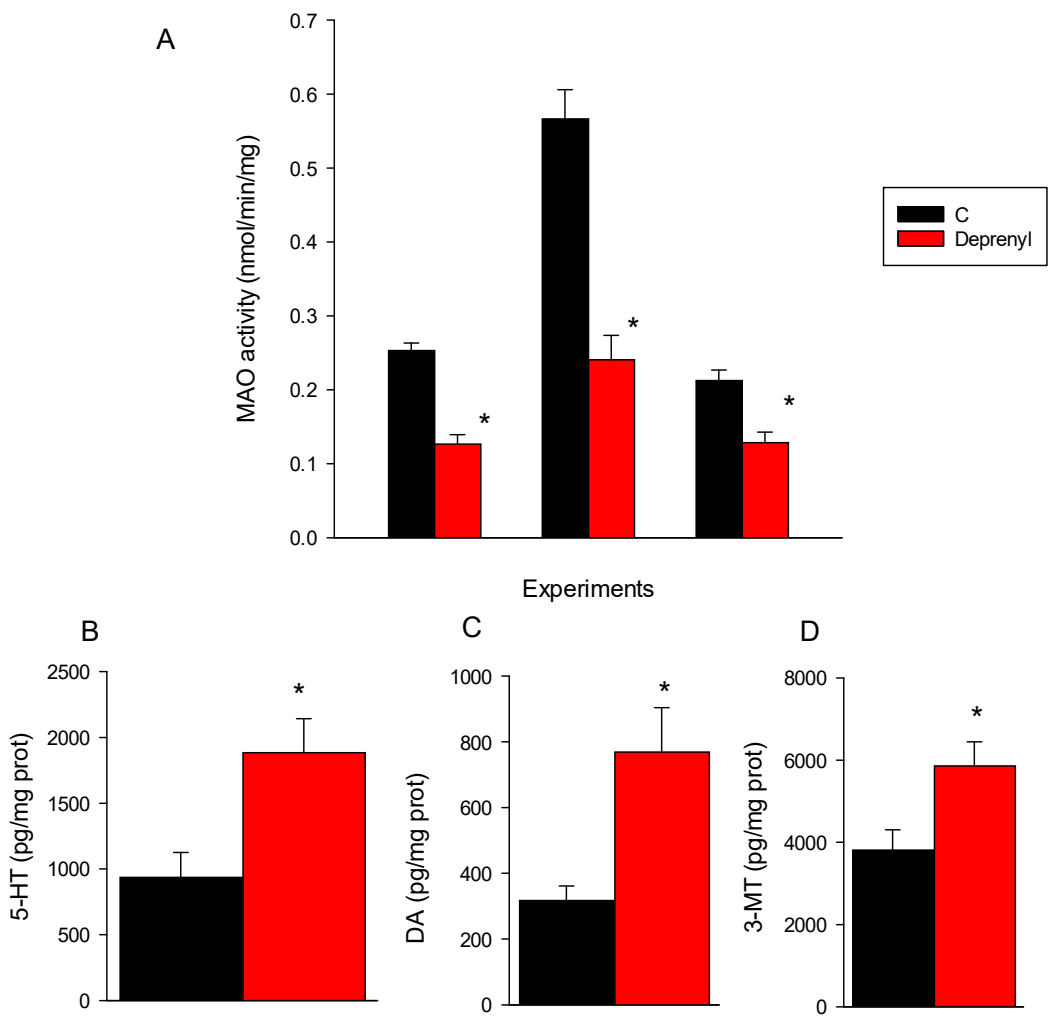

Figure 3. Effects over enzyme activity (A) and monoaminergic neurotransmitter levels (B-D) (means $\pm \mathrm{SE}, n=5-10$ ) in the whole body of the $D$. magna juveniles following 24 exposures to $1 \mathrm{mg} / \mathrm{L}$ of deprenyl. For clarity, monoaminergic neurotransmitters that showed a significant change across treatments are depicted. The rest of the values are provided in Table S3, Supplementary Materials. Note: * the indicated bars mean significant $(p<0.05)$ treatment differences relative to the controls following the Student's $t$-test. 


\section{Discussion}

In this study, we provided for the first time the results on the mode of action of deprenyl in D. magna. Deprenyl at $1 \mathrm{mg} / \mathrm{L}$ inhibited about $50 \%$ of the MAO activity and by doing so increased the concentrations of serotonin and dopamine by 2- and 2.4fold, respectively. There are two types of MAO inhibitors, type A and type B. Type A inhibitors block the catabolism of noradrenaline and serotonin, and type $\mathrm{B}$ inhibitors block the catabolism of dopamine. Deprenyl is a MAO type B inhibitor in mammalian models; however, at high dosages, it inhibits both type A and type B MAO [15]. This means that deprenyl, apparently, has a similar mechanism of action as in vertebrates (i.e., mammals and fish) inhibiting MAO type B but also A activity and thus preventing the metabolism of serotonin and dopamine $[14,16]$. There is controversy on the role, if any, of the MAO types on the metabolism of monoamines in arthropods [17]. According to the previous review, alternative metabolic routes such as $N$-acetylation, $\gamma$-glutamyl conjugation, sugar conjugation, sulfation, $\beta$-alanyl conjugation are predominantly used by insects and crustaceans to metabolize monoamines. The reported evidence, however, indicates that ticks and mites have a unique MAO sensitive to deprenyl that catabolizes serotonin and dopamine [18,19]. Furthermore, in the hepatopancreas of the crab Paralithodes camtschaticus, there is also only one type of MAO that shows great inhibition specificity for deprenyl [20]. The D. magna genome contains a unique flavin-containing monoamine oxidase A gene/protein (ACC XP_032781527.1) that has 54\% homology with a putative MAO protein of the shrimp Penaeus vannamei (XP_027230105.1), 33\%—with human MAOs. These homologies seem moderate but note that the zebrafish MAO gene/protein has only $69 \%$ homology with those of humans. Thus, the results obtained in this study are in line with the previously reported studies on ticks, mites and crabs and provide evidence for the presence of a MAO-like activity in D. magna that is able to metabolize serotonin and dopamine.

Using similar concentrations of deprenyl ( $5 \mu \mathrm{M} \cong 0.94 \mathrm{mg} / \mathrm{L})$, Faria et al. [14] reported similar background activities of MAO in zebrafish larvae, but an almost complete inhibition of MAO activity and a greater increase of serotonin than of dopamine. Phylogenetic differences between $D$. magna, fish and mammals are likely to account for the observed deprenyl specificity effect on the MAO activity and of the latter enzyme for catabolising serotonin and dopamine [21]. Deprenyl, despite decreasing the MAO activity and enhancing serotonin, did not decrease the serotonin degradation metabolite 5-hydroxyindoleacetic acid (5-HIAA). There are, however, reported discrepancies on the consequences of the MAO type A,B inhibition on serotonin degradation metabolites. The reported studies on the zebrafish exposed to deprenyl found enhanced or unchanged levels of 5-HIAA [14,22]. In rodents, MAO type A inhibition that lead to enhanced levels of serotonin did not necessarily affect its metabolite 5-HIAA [23].

Deprenyl increased, however, the concentration of the dopamine metabolite 3methoxytyramine (3-MT) in D. magna, an effect previously reported for mice treated with the MAO type A inhibitor clorgyline [24].

In a previous study we found that neuroactive drugs do not always show the same target specificity in D. magna as in mammals [13]. In relation to this, we analysed up to 16 metabolites belonging to four neurological metabolic pathways (i.e., serotonin, catecholamine, cholinergic and GABAergic). The results obtained for deprenyl showed high specificity for its putative serotonergic and dopaminergic targets.

The study of behavioural responses indicates contrasting effects of deprenyl against compounds that are known that decrease serotonin in Daphnia such as PCPA [13]. Deprenyl increased habituation to repetitive light stimuli, which is a primary form of non-associative learning [25], and enhanced responses to light in $50 \%$ of the experiments performed (1 out of 4 in Figure 1; 3 out of 4 in Figure 2). Conversely, PCPA-treated organisms took longer to habituate to the repetitive light stimuli and responded to a lower extent to visual light stimuli. Interestingly, D. magna individuals exposed to deprenyl also had a lower basal activity, which was monitored under darkness. The behavioural features of PCPA are 
consistent with the reported higher basal activity and reduced habituation of CRISPRmediated TPH-mutated D. magna juveniles that lack serotonin [11]. In zebrafish larvae exposed to deprenyl, Faria et al. [14] also reported a lower basal activity, a reduced response to light or tactile stimuli and increased habituation. In fish and also in mammals, increased serotonin levels have sedative-like anxiolytic effects [14,26], whereas in D. magna, it is unclear. The results obtained here in D. magna for deprenyl apparently agree with those of zebrafish [14] since in both species it decreases the basal locomotor activity and increases habituation. However, in fish, deprenyl also decreased the response to stimuli [14], which agrees with the reported decreased anxiety-like responses in rodents [26]. In D. magna, deprenyl either did not affect or enhanced the response to stimuli. In relation to this, it is important to remark that, unlike in zebrafish, deprenyl increased the dopamine level to the same extent as that of serotonin. Increased dopamine levels have been associated with a hyperresponsive behaviour to mechanical stimuli in Drosophila melanogaster and Caenorhabditis elegans [27]. Of course, dopamine does not act alone in regulating behavioural responsiveness to stimuli; there are counteracting neuronal systems, such as serotonin and other monoamines $[27,28]$. Therefore, the observed behavioural defects in the D. magna exposed to deprenyl are likely to be related to the observed enhanced levels of dopamine and serotonin.

In fish and also in mammals, decreasing serotonin levels induced by PCPA cause anxiety-like behaviour such as hyperlocomotion activity and enhanced responses to stimuli [14,29]. PCPA is also known to impair learning [30]. The D. magna exposed to PCPA showed basal hyperactivity only in one out of the two experiments, increased the response to light in some of the trials performed, but impaired learning in all the trials (decreased habituation). This means that the D. magna responses to PCPA can be related to anxiogenic behaviour only in part. Nevertheless, the previous results obtained with knockout D. magna lacking serotonin did show higher hyperactivity, enhanced responses to light stimuli and also impaired habituation $[2,11]$, a phenotype compatible with anxiety-like behaviour and learning impairment in rodents [30,31]. The apparently closer phenotype of genetically modified D. magna individuals lacking serotonin than of those enzymatically impaired by PCPA can be related to the unspecific action of the latter compound. PCPA not only reduced serotonin in D. magna but also decreased the levels of norepinephrine [13], which is known to modulate arousal and other types of cognitive behaviour [32].

\section{Conclusions}

The results reported here show that D. magna juveniles are sensitive to MAO inhibitors that change serotonin signalling. In addition, molecular targets of MAO modulators such as effects on the enzymatic activity and on the concentration of serotonergic and dopaminergic metabolites was also observed. The model serotonin modulator deprenyl inhibited the MAO activity and increased the serotonin, dopamine and dopamine metabolite 3-MT levels. The deprenyl-treated individuals showed consistently shorter habituation to repetitive light stimuli and reduced basal locomotor activity. Deprenyl behavioural outcomes opposed those of the PCPA drug or genetically modified individuals having reduced serotonin levels. The findings presented in this study reinforce the use of this nonvertebrate model to address behavioural and physiological roles of serotonin.

Supplementary Materials: The following are available online at https:/ / www.mdpi.com/article/10 $.3390 /$ toxics 9080187 /s1, Supplementary Methods and Supplementary Results-Tables. Table S1: List the metabolites analysed, Table S2. Quality parameters of monoamine neurotransmitters and the related metabolites, Table S3: Student's t-test results for MAO and monoaminergic neurotransmitter levels, Table S4: List of values for the nonsignificant metabolites.

Author Contributions: Conceptualization, M.B., D.R. and C.B.; Methodology, M.B. and M.F.; Validation, M.B.; Investigation, M.B.; Formal analysis, M.B. and C.B.; Writing—original draft preparation, M.B. and C.B.; Writing-review and editing, M.B., C.G.-C., D.R. and C.B.; Supervision, C.G.-C. and C.B.; Visualization, C.B. All authors have read and agreed to the published version of the manuscript. 
Funding: This work was supported by "Agencia Estatal de Investigación" from the Spanish Ministry of Science and Innovation (project PID2020-113371RB-C21), IDAEA-CSIC, Severo Ochoa Centre of Excellence (CEX2018-000794-S), which financed M.F. with Severo Ochoa funds. The work was partially supported by the Catalan Government through the network of recognized research groups (2017 SGR_902).

Institutional Review Board Statement: Not applicable.

Informed Consent Statement: Not applicable.

Data Availability Statement: Data supporting the reported results will be provided upon reader's request.

Conflicts of Interest: The authors declare no conflict of interest.

\section{References}

1. Burks, R.L.; Jeppesen, E.; Lodge, D.M. Littoral zone structures as Daphnia refugia against fish predators. Limnol. Oceanogr. 2001, 46, 230-237. [CrossRef]

2. Bedrossiantz, J.; Martínez-Jerónimo, F.; Bellot, M.; Raldua, D.; Gómez-Canela, C.; Barata, C. A high-throughput assay for screening environmental pollutants and drugs impairing predator avoidance in Daphnia magna. Sci. Total Environ. 2020, 740. [CrossRef]

3. Faria, M.; Prats, E.; Novoa-Luna, K.A.; Bedrossiantz, J.; Gómez-Canela, C.; Gómez-Oliván, L.M.; Raldúa, D. Development of a vibrational startle response assay for screening environmental pollutants and drugs impairing predator avoidance. Sci. Total Environ. 2019, 650, 87-96. [CrossRef] [PubMed]

4. Berger, M.; Gray, J.A.; Roth, B.L. The expanded biology of serotonin. Annu. Rev. Med. 2009, 60, 355-366. [CrossRef] [PubMed]

5. O'Mahony, S.M.; Clarke, G.; Borre, Y.E.; Dinan, T.G.; Cryan, J.F. Serotonin, tryptophan metabolism and the brain-gut-microbiome axis. Behav. Brain Res. 2015, 277, 32-48. [CrossRef]

6. Busch, W.; Schmidt, S.; Kühne, R.; Schulze, T.; Krauss, M.; Altenburger, R. Micropollutants in European rivers: A mode of action survey to support the development of effect-based tools for water monitoring. Environ. Toxicol. Chem. 2016, 35, 1887-1899. [CrossRef]

7. Fong, P.P.; Ford, A.T. The biological effects of antidepressants on the molluscs and crustaceans: A review. Aquat. Toxicol. 2014, 151, 4-13. [CrossRef] [PubMed]

8. Brooks, B.W.; Foran, C.M.; Richards, S.M.; Weston, J.; Turner, P.K.; Stanley, J.K.; Solomon, K.R.; Slattery, M.; La Point, T.W. Aquatic ecotoxicology of fluoxetine. Toxicol. Lett. 2003, 142, 169-183. [CrossRef]

9. Orsini, L.; Gilbert, D.; Podicheti, R.; Jansen, M.; Brown, J.B.; Solari, O.S.; Spanier, K.I.; Colbourne, J.K.; Rush, D.; Decaestecker, E. Daphnia magna transcriptome by RNA-Seq across 12 environmental stressors. Sci. Data 2016, 3, 1-16. [CrossRef]

10. Gunnarsson, L.; Jauhiainen, A.; Kristiansson, E.; Nerman, O.; Larsson, D.G.J. Evolutionary conservation of human drug targets in organisms used for environmental risk assessments. Environ. Sci. Technol. 2008, 42, 5807-5813. [CrossRef] [PubMed]

11. Rivetti, C.; Campos, B.; Piña, B.; Raldúa, D.; Kato, Y.; Watanabe, H.; Barata, C. Tryptophan hydroxylase (TRH) loss of function mutations induce growth and behavioral defects in Daphnia magna. Sci. Rep. 2018, 8, 11. [CrossRef] [PubMed]

12. Campos, B.; Rivetti, C.; Kress, T.; Barata, C.; Dircksen, H. Depressing Antidepressant: Fluoxetine Affects Serotonin Neurons Causing Adverse Reproductive Responses in Daphnia magna. Environ. Sci. Technol. 2016, 50. [CrossRef]

13. Fuertes, I.; Barata, C. Characterization of neurotransmitters and related metabolites in Daphnia magna juveniles deficient in serotonin and exposed to neuroactive chemicals that affect its behavior: A targeted LC-MS/MS method. Chemosphere 2021, 263, 127814. [CrossRef] [PubMed]

14. Faria, M.; Prats, E.; Bellot, M.; Gomez-Canela, C.; Raldúa, D. Pharmacological modulation of serotonin levels in zebrafish larvae: Lessons for identifying environmental neurotoxicants targeting the serotonergic system. Toxics 2021, 9, 118. [CrossRef] [PubMed]

15. Huber, S.J.; Cummings, J.L. Parkinson's Disease: Neurobehavioral Aspects; Oxford University Press: Oxford, UK, 1992; ISBN 0195069692.

16. Riederer, P.; Youdim, M.B.H.; Rausch, W.D.; Birkmayer, W.; Jellinger, K.; Seemann, D. On the mode of action of L-deprenyl in the human central nervous system. J. Neural Transm. 1978, 43, 217-226. [CrossRef]

17. Sloley, B.D. Metabolism of Monoamines in Invertebrates: The Relative Importance of Monoamine Oxidase in Different Phyla. Neurotoxicology 2004, 25, 175-183. [CrossRef]

18. Kadir, H.A.; Knowles, C.O. Oxidative deamination and N-acetylation of biogenic amines by homogenates of bulb mites (Rhizoglyphus echinopus). Comp. Biochem. Physiol. Part C Comp. 1989, 94, 465-468. [CrossRef]

19. Kaufman, R.; Sloley, D. Catabolism of dopamine and 5-hydroxytryptamine by monoamine oxidase in the ixodid tick, Amblyomma hebraeum. Insect Biochem. Mol. Biol. 1996, 26, 101-109. [CrossRef]

20. Basova, N.E.; Basova, I.N.; Yagodina, O. V Monoamine Oxidase Activity in the Hepatopancreas of the Kamchatka Crab Paralithodes camtschaticus: A Substrate-Inhibitor Specificity. J. Evol. Biochem. Physiol. 2018, 54, 345-352. [CrossRef]

21. Setini, A.; Pierucci, F.; Senatori, O.; Nicotra, A. Molecular characterization of monoamine oxidase in zebrafish (Danio rerio). Comp. Biochem. Physiol. B Biochem. Mol. Biol. 2005, 140, 153-161. [CrossRef] 
22. Sallinen, V.; Sundvik, M.; Reenilä, I.; Peitsaro, N.; Khrustalyov, D.; Anichtchik, O.; Toleikyte, G.; Kaslin, J.; Panula, P. Hyperserotonergic phenotype after monoamine oxidase inhibition in larval zebrafish. J. Neurochem. 2009, 109, 403-415. [CrossRef] [PubMed]

23. Finberg, J.P.M.; Rabey, J.M. Inhibitors of MAO-A and MAO-B in psychiatry and neurology. Front. Pharmacol. 2016, 7. [CrossRef] [PubMed]

24. Kato, T.; Dong, B.; Ishii, K.; Kinemuchi, H. Brain Dialysis: In Vivo Metabolism of Dopamine and Serotonin by Monoamine Oxidase A but Not B in the Striatum of Unrestrained Rats. J. Neurochem. 1986, 46, 1277-1282. [CrossRef] [PubMed]

25. Kandel, E.R. Cellular mechanisms of learning and the biological basis of individuality. Princ. Neural Sci. 1991, 3, $1009-1031$.

26. Bortolato, M.; Godar, S.C.; Davarian, S.; Chen, K.; Shih, J.C. Behavioral disinhibition and reduced anxiety-like behaviors in monoamine oxidase b-deficient mice. Neuropsychopharmacology 2009, 34, 2746-2757. [CrossRef]

27. Van Swinderen, B.; Andretic, R. Dopamine in Drosophila: Setting arousal thresholds in a miniature brain. Proc. R. Soc. B Biol. Sci. 2011, 278, 906-913. [CrossRef]

28. Fischer, A.G.; Ullsperger, M. An update on the role of serotonin and its interplay with dopamine for reward. Front. Hum. Neurosci. 2017, 11. [CrossRef]

29. Kshama, D.; Hrishikeshavan, H.J.; Shanbhogue, R.; Munonyedi, U.S. Modulation of baseline behavior in rats by putative serotonergic agents in three ethoexperimental paradigms. Behav. Neural Biol. 1990, 54, 234-253. [CrossRef]

30. Izquierdo, A.; Carlos, K.; Ostrander, S.; Rodriguez, D.; McCall-Craddolph, A.; Yagnik, G.; Zhou, F. Impaired reward learning and intact motivation after serotonin depletion in rats. Behav. Brain Res. 2012, 233, 494-499. [CrossRef]

31. Waider, J.; Popp, S.; Lange, M.D.; Kern, R.; Kolter, J.F.; Kobler, J.; Donner, N.C.; Lowe, K.R.; Malzbender, J.H.; Brazell, C.J.; et al. Genetically driven brain serotonin deficiency facilitates panic-like escape behavior in mice. Transl. Psychiatry 2017, 7, e1246. [CrossRef]

32. Mather, M.; Clewett, D.; Sakaki, M.; Harley, C.W. Norepinephrine ignites local hotspots of neuronal excitation: How arousal amplifies selectivity in perception and memory. Behav. Brain Sci. 2016, 39. [CrossRef] [PubMed] 